\title{
Reducing Maternal Mortality from Direct Obstetric Causes during 2013 in Sudan
}

\author{
Umbeli Taha $^{1}$, Salah Ismail ${ }^{1}$, Elmahgoub AbdAlla ${ }^{1}$, Kunna Abd Alilah ${ }^{2}$, Sawsan Eltahir $^{3}$ and Salman Mirghani ${ }^{4}$ \\ 1. Department of Obstetrics and Gynecology, Omdurman Islamic University (OIU), Omdurman, Khartoum, Sudan \\ 2. Department of Obstetrics and Gynecology, University of Bahri, Khartoum North, Khartoum, Sudan. \\ 3. Reproductive Health Program, Federal Ministry of Health, Khartoum, Sudan \\ 4. Department of Obstetrics and Gynecology, Omdurman Maternity Hospital, Omdurman, Sudan
}

\begin{abstract}
Despite the worldwide commitment to improve maternal health, measuring, monitoring and comparing mortality estimates remain a challenge. This study was done to assess maternal mortality from direct obstetric causes (obstetric haemorrhage, hypertensive disorders and sepsis) after suitable interventions in Sudan during 2013. Facility and community-based MDR (maternal death review) was conducted in Sudan since 2009. National and states' MDRCs (Maternal Death Review Committees) were established. A focal person for each state, health facility and locality was nominated. Notification of MD (maternal deaths) was done by telephone, followed by review of all notified maternal deaths using a structured format. Recommendations for reducing MM (maternal mortality) from obstetric haemorrhage, hypertensive disorders and sepsis have been generated. Management protocols, training of care providers and availing of necessary requirements for these cases have been approved and implemented in all states. Reported MD were reviewed and analyzed using SPSS, version 18.0 and the resulting MMR (maternal mortality ratio) was compared with years 2010-2012 to assess improvement. During 2013, 1,110 maternal deaths were notified, out of 645,881 LB (live births). MMR was 172/100,000 LB, with different variation between states. Facility maternal deaths were 937 (84.4\%) and community deaths were 173 (15.6\%). Direct obstetric deaths were $678(61.1 \%)$, mainly due to haemorrhage 311 (28.0\%), hypertensive disorders $116(10.5 \%)$ and sepsis $110(9.9 \%)$. Indirect causes were 432 (38.9\%), mainly due to hepatitis and anemia. Most of the hospital deaths 707 (75.5\%) were admitted late from home and 559 (59.7\%) died within 24 hours. MDs from hypertensive disorders and sepsis decreased significantly, while MDs from haemorrhage are persistently high compared to 2010-2012. Home delivery, late presentation, unavailability of blood and poor referral system, are the main factors behind maternal deaths. MDs from hypertensive disorder and sepsis decreased significantly, while MDs from haemorrhage are persistently high.
\end{abstract}

Key words: Maternal mortality, maternal death review, direct obstetric causes, Sudan.

\section{Introduction}

Maternal deaths and disabilities remain major public health problem in the developing countries. In order to end preventable maternal deaths, accurate information on how many women died, where they died and how they died is essential, but it is currently inadequate [1]. A major challenge to achieving MDG5 (Millennium Development Goal 5) by developing countries is the lack of reliable and valid information on maternal deaths and disabilities on which to measure progress

Corresponding author: Umbeli Taha, Ph.D., research fields: obstetrics and gynecology, women health research. E-mail: umbeli_taha@hotmail.com. made towards its attainment [2]. MDR (maternal death review) is a key element of a strategy to improve the quality of maternal healthcare by determining the causes of deaths and recommending intervention to avert each death and improve the quality of care based on the findings. Reporting of maternal death is an important element of health management information systems as long as it leads to the allocation of resources and taking responsibility for actions to prevent avoidable deaths [3].

In Sudan, like many African countries there are documented successes in reducing maternal death, improving quality of care, and increasing client 
satisfaction and use of available services [4]. MDR had been introduced since 2009, with regular annual report: identifying the causes of maternal deaths and areas of delay. Suggested recommendations have been generated, and many interventions are now implemented to reduce MMR (maternal mortality ratio) from direct causes including: haemorrhage, hypertensive disorder and sepsis. This study aims at assessing MMR reduction from hemorrhage, hypertensive disorder and sepsis during 2013 in Sudan.

\section{Materials and Methods}

This is a descriptive, facility and community-based study, conducted in Sudan, in the year 2013. All women died in hospital or community from pregnancy related conditions were included. A structured questionnaire was used and a more detailed questionnaire related to specific condition, including haemorrhage, sepsis, hypertensive disorder and hepatitis was used to determine gaps and delay in management received [5]. Data was collected by trained registrars and health care providers. A one day workshop has been conducted, which was attended by obstetricians, registrars in obstetrics and gynaecology and administrative personnel to endorse and disseminate the national proposal for maternal mortality reviews. Received forms were indexed, reviewed, classified into avoidable and non-avoidable maternal mortality, and were edited by trained personnel. Edited data was rechecked for accuracy, by picking $10 \%$ of the received forms. Data was analyzed using SPSS version 18.0, and results were discussed with the NMDRC (National Maternal Death Review Committee). Guidelines for management of obstetric haemorrhage, hypertensive disorder and sepsis have been designed by expert committee and approved by the council of obstetrics and gynecology, endorsed in a workshop including all stakeholders and implemented at all hospital levels with availing of all necessary requirements and training of health care providers, including midwives. Supportive supervision was carried out for checking adherence to guidelines, availability of required supplies and implementation of intervention.

\section{Results}

The results highlight the size of the problem and common causes of maternal death in 2013 compared to 2010-2012. Total number of notified maternal deaths was 1,110 , out of $645,881 \mathrm{LB}$ (live births) (Table 1).

Table 1 Distribution of MD and MMR according to state from 2012-2013.

\begin{tabular}{lllllll}
\hline State & Maternal death 2012 & Live birth & MMR 2012 & Maternal death 2013 & Live birth & MMR 2013 \\
\hline Khartoum State & 165 & 171,976 & 96 & 175 & 182,930 & 96 \\
Gazira & 102 & 80,740 & 126 & 90 & 88,590 & 102 \\
River Nile & 27 & 28,055 & 96 & 17 & 16,313 & 104 \\
Gadaref & 58 & 49,251 & 118 & 51 & 46,331 & 110 \\
N. State & 19 & 12,337 & 154 & 14 & 11,985 & 117 \\
Kasala & 91 & 42,310 & 215 & 79 & 65,400 & 121 \\
S. Darfur & 33 & 13,823 & 239 & 56 & 33,147 & 169 \\
White Nile & 71 & 31,506 & 225 & 53 & 30,866 & 172 \\
W.n Darfur & 123 & 24,551 & 501 & 41 & 23,814 & 172 \\
Sennar & 54 & 25,262 & 214 & 069 & 25,153 & 274 \\
Red Sea & 44 & 15,691 & 268 & 49 & 16,014 & 305 \\
Blue Nile & 54 & 22,952 & 235 & 83 & 26,234 & 316 \\
N. Darfur & 76 & 18,557 & 410 & 84 & 22,727 & 370 \\
S. Kurdofan & 85 & 20,462 & 415 & 89 & 21,680 & 411 \\
N. Kurdofan & 92 & 26,246 & 351 & 132 & 26,630 & 496 \\
Central Darfur & - & - & - & 28 & 8,430 & 332 \\
Sudan & 1,094 & 583,719 & 187 & 1,110 & 646,244 & 172
\end{tabular}






Fig. 1 MMR according to year 2010-2013.

Table 2 Distribution of cause of death.

\begin{tabular}{lll}
\hline Cause of death & Frequency & Percent \\
\hline Obstetric haemorrhage & 311 & $28.0 \%$ \\
Hepatitis & 182 & $16.4 \%$ \\
Eclampsia & 116 & $10.5 \%$ \\
Sepsis & 110 & $9.9 \%$ \\
Anemia related conditions & 61 & $5.5 \%$ \\
Ruptured uterus & 56 & $5.0 \%$ \\
Abortion & 43 & $3.9 \%$ \\
Malaria & 35 & $3.2 \%$ \\
Anesthesia & 25 & $2.3 \%$ \\
PE (pulmonary embolism) & 17 & $1.5 \%$ \\
Febrile illness & 4 & $0.4 \%$ \\
Unclassified* & 150 & $13.4 \%$ \\
Total & 1,110 & $100.0 \%$ \\
\hline
\end{tabular}

*APE (amniotic fluid embolism), ARF (acute renal failure), cerebral infarction, CVA (cerebro vascular accidents), lung fibrosis, PUO (pyrexia of unknown origin), status asthmaticus, Gallium Bare, DKA (diabetic keto-acidosis) and dengue fever.

MMR is $172 / 100,000$ LB (Fig. 1). Institutional maternal deaths were 937 cases $(84.4 \%)$ and maternal deaths at community were 173 cases (15.6\%). From this analysis, $701(63.2 \%)$ were classified as avoidable deaths and 409 (36.8\%) were unavoidable. Among hospital deaths, 559 cases (59.4\%) died within the first $24 \mathrm{~h}$ from admission, and most of them were avoidable and due to first and second delay. 707 (75.5\%) of the women died in hospital were admitted as emergency from home bypassing nearby health facilities, only 175 (15.8\%) were referred from other health facilities. Maternal deaths due to direct causes were 678 (61.1\%), MD (maternal deaths) due to haemorrhage was 311
(28.0\%), hypertensive disorder was $116(10.5 \%)$ and sepsis was 110 (9.9\%). Indirect MDs were 432 (38.9\%), mostly due to hepatitis and anemia related conditions.

Maternal death from obstetric haemorrhage was 311 cases $(28.0 \%)$. PPH (post partum haemorrhage) was 252 (81.1\%), APH (ante-partum haemorrhage) was 58 $(18.6 \%)$ and one case $(0.3 \%)$ was due to blood disorder (Table 2). PPH, which is mainly due to uterine atonia was $140(55.5 \%)$, retained placenta was $42(16.7 \%)$, ruptured uterus was $56(22.2 \%)$ and birth canal injuries was 14 (5.6\%). Only 129 (41.5\%) delivered at home, and $83(26.7 \%)$ died at home or on arrival. Among patients admitted to hospital, 107 (46.9\%) received 
blood transfusion. Blood was not available, due to absence of donors, which was 30 (24.8\%), or a non-functioning blood bank which was 54 cases (44.6\%). All admitted patients received Uterotonics, except three $(1.3 \%)$.

Maternal death from hypertensive disorder, ante partum eclampsia and post partum eclampsia was 116 (10.5\%), $76(65.5 \%)$ and $27(23.3 \%)$, respectively. There were six cases $(5.2 \%)$ of intrapartum eclampsia and seven cases $(6.0 \%)$ with severe pre-eclampsia. In 92 cases $(79.3 \%)$, the first fit started at home and five $(4.3 \%)$ of them died there. Ninety six cases (59.5\%) died within $24 \mathrm{~h}$ from onset of the first fit. Sixty one (52.6\%) had five or more fits before they died. CVA (cerebro vascular accidents) was the common reported cause of maternal death 69 (59.5\%), followed by haemorrhage which was $16(13.8 \%)$, acute pulmonary edema was $13(11.2 \%)$, acute renal failure was 10 $(8.6 \%)$, while air way obstruction was eight cases (6.9\%). Magnesium sulfate was not used in 27 cases $(24.3 \%)$, either because it was not available, no protocol for its use or it is not used at all in that hospital and fifty two cases $(46.8 \%)$ did not find access for ICU (intensive care unit).

Maternal deaths from sepsis were 110 (9.9\%), 48 (43.6\%) delivered by Em C/S (emergency cesarean section), vaginal delivery at home was 39 (35.5\%), instrumental vaginal delivery was 12 (10.9\%), incomplete abortion was $10(9.1 \%)$, while one case was by elective $\mathrm{C} / \mathrm{S}$. In 58 cases $(52.8 \%)$ the first stage was more than $24 \mathrm{~h}$, however, the second stage was more than $2 \mathrm{~h}$ in 59 cases $(53.6 \%)$. They all died in the hospital, mainly due to septicemia with 108 (98.2\%) cases and in 95 cases $(86.4 \%)$ no intervention was taken to know the source or causative agent of infection (Fig. 2).

\section{Discussion}

To achieve MDG5 and reduce the maternal mortality ratio by three-quarters before 2015, improving health care for women and providing universal access to reproductive health services must be prioritized. This includes, availing of accurate data for intervention, access to family planning, prevention of unplanned pregnancies, provision of high-quality pregnancy and delivery care, including emergency obstetric care. Sudan has scarce data, however, there is currently increasing interest in directing the limited resources into efforts to understand why maternal death persists and what can be done to avert maternal deaths and disability.

In this report of MDR, there is significant increase in the number of notified MD, completeness of review forms and eventually reduction in MMR country wide

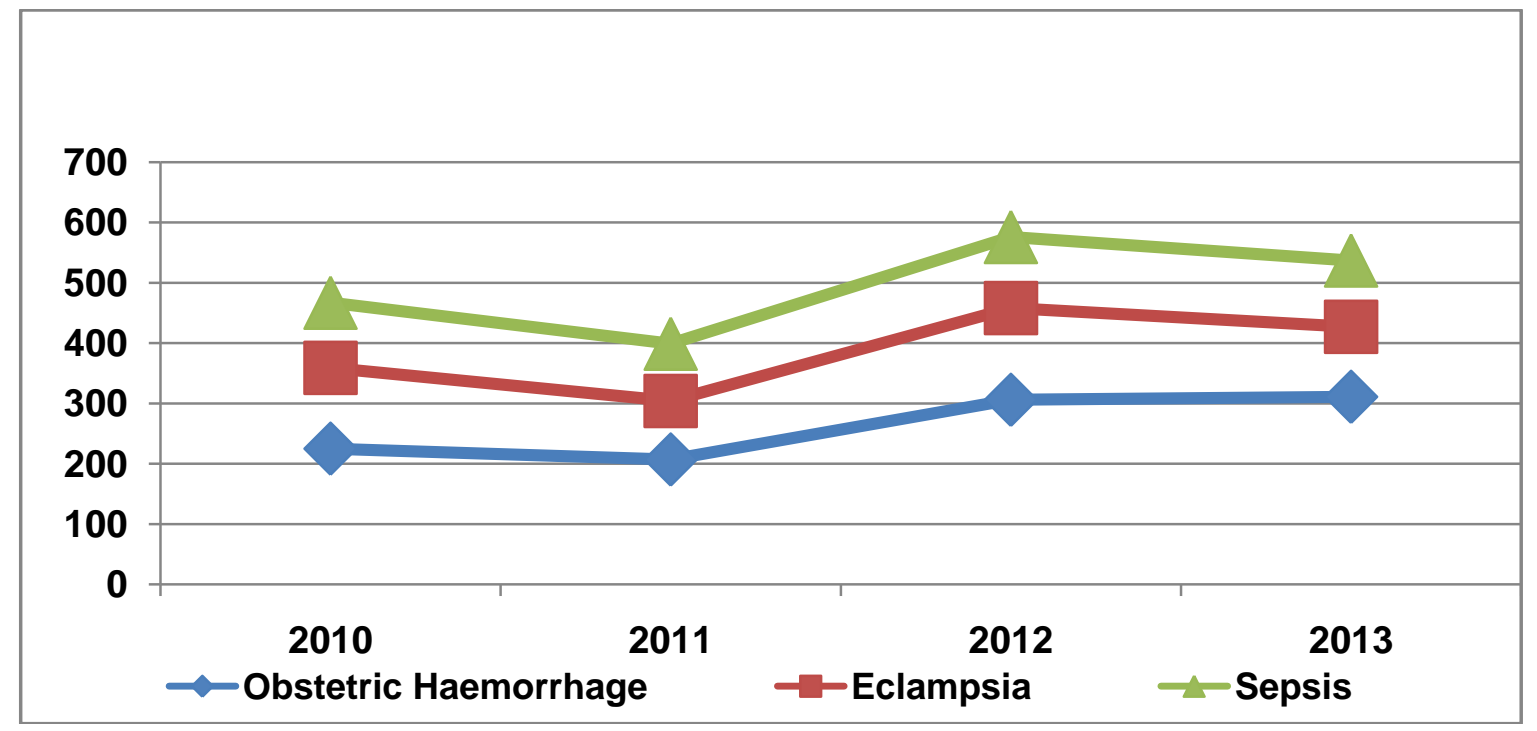

Fig. 2 Trend in causes of MD 2010-2013. 
and in particular states. The high number of maternal deaths in some states reflects inequities in access to health services, and highlights the gap between the rich and the poor, rural and urban citizens. Poor women in remote areas are the least likely to receive adequate health care. This is especially true for states with low numbers of skilled health workers, such as Darfur states. While levels of antenatal care have increased in many parts of the country during the past decade, only $46 \%$ of women benefit from skilled care during childbirth. This means that a considerable number of births are not assisted by a midwife, a doctor or a trained nurse. In-service training, motivation and monitoring are necessary urgent intervention to improve the situation. This MMR is comparatively lower than MMR reported in 2010, 2011 and 2012, as well as that found in district hospital in Senegal $[6,7]$. It is even lower compared to DHS (demographic health survey), SMS (safe motherhood survey), SHHS (Sudan house hold survey) 2006 and 2010, however, it is still higher than our target MDG5, 124/100,000 LB [6]. It is clear that late presentation to hospital, where 750 (67.6\%) were admitted critically ill and $559(59.7 \%)$ of them died within the first $24 \mathrm{~h}$, are important etiological factors behind maternal deaths.

The first and second delays are major determinants of MD in this report. Many efforts have been made to raise community awareness towards $\mathrm{MD}$ and utilization of available resources. ANC (improving antenatal care), SAB (skilled attendance at birth) and referral system in remote areas will improve access to health care and reduce maternal mortality. As seen in this report, only 175 (15.3\%) were referred cases and were too late to save their lives. Progress in reduction of MMR is happening especially in states where all stakeholders including the UN, donors, care providers and civil society are working together effectively to create real change for women and children health. Maternal mortality has started decreasing in states where intervention were properly implemented, e.g., Khartoum, Gazira, White Nile, River Nile, Northern
State, Gadarif, and Kassala. Regional or states variation may be due to concentration of obstetric services in certain states, with more socio-demographic factors adversely affecting MMR in other states and good implementation of interventions in others.

MD from direct obstetric causes was 678 (61.1\%), which is relatively low compared to WHO (World Health Organization) reports, of $73 \%$ of all MD [8]. Maternal deaths from obstetric haemorrhage, hypertensive disorder and sepsis was 537 (48.4\%) of all $\mathrm{MD}$, this is consistent with that found by WHO, where it is more than half of MD worldwide [8]. Hemorrhage is the leading cause of death in this review $311(28.0 \%)$, and it is comparable to that found by WHO (27.1\%) worldwide and in developing countries [8]. It is still high particularly from PPH $252(81.1 \%)$, which is mainly due to atonia $140(55.5 \%)$, retained placenta and ruptured uterus. All of them can be reduced by AMTSL (active management of third stage of labour) and proper intra-partum care. The implementation of guidelines and adherence to management protocols will improve obstetric performance, together with availability of requirements, including drugs, blood and referral system to reduce maternal mortality. Home delivery $129(41.5 \%)$ is an important etiological factor, which can be reduced by improving SAB (skilled attendance at birth), increasing institutional delivery and good referral system. Lack of blood for various unacceptable reasons is also an important leading factor for MD resulting from haemorrhage. Even in developed countries, hemorrhage is still among the main causes of maternal morbidity [9]. According to WHO analysis, hemorrhage constitutes $33.9 \%$ of maternal deaths in Africa and 30.8\% in Asia [10]. In both developed and developing countries, $60 \%$ of maternal mortality occurred in the post partum period, $45 \%$ occurred during the first day of delivery, $65 \%$ within the first week and $80 \%$ within two weeks [11].

Maternal death from hypertensive disorders are relatively low in this review, $116(10.5 \%)$, compared to 
2011, 11.3\% and 2012, 13.8\% [12]. It is consistent with that found by WHO, $11.1 \%$ worldwide, $14.0 \%$ in developing countries and $16.0 \%$ in Sub-Saharan Africa [8]. According to WHO's analysis, $25.7 \%$ of MD in Latin America and Caribbean were due to hypertensive disorders [13]. However, it is unacceptably high in Khartoum state, 25 (21.6\%), in spite of the available facilities, staff, drugs, high dependency units, guidelines and treatment protocols. It may be due to increased number of referred complicated cases from rural areas. This low level of MD from hypertensive disorder may be due to the implementation of guidelines for use of magnesium sulphate for the treatment of eclampsia and prevention of onset of eclampsia in severe pre-eclampsia as well as training of care providers for use and assessment of its side effects. Strengthening of implementation, availing of drugs, improving ANC and referral system will reduce MMR from hypertensive disorders.

Maternal deaths from sepsis was 110 (9.9\%), which is consistent with that found by WHO, $10.7 \%$ worldwide and $10.3 \%$ in Sub-Saharan Africa [8]. This reduction may be due to increased number of trained midwives and improvement in safety measures for reducing infection during labour and peurperium, together with prophylactic use of antibiotics for group of patients at risk.

\section{Conclusion}

This review may accelerate actions to remove existing obstacles to progress and can make a significant change in the remaining two years to meet the MDG targets. Home delivery, late presentation, late intervention, unavailability of blood and its requirements, and poor referral system are the main factors behind MD. MD from hypertensive disorder and sepsis decreased significantly, while MD from haemorrhage is persistently high. These results should inform health policies and programmers for prioritization and funding to reduce maternal mortality.

Maternal death challenges are mainly due to administrative and inadequate resources, poor budget, limited untrained staff, frequent turnover and limited awareness on the importance of notification of MD and reporting of live birth.

\section{Acknowledgment}

Authors would like to thank ministry of higher education and research for sponsoring this research, UNFPA Sudan office for their appreciated efforts in MDR, and particularly, their inputs in their target states. Our thanks are extended to UNICEF, Sudan office for their support in conducting supportive supervision tours and NMDRC meetings. Thanks are extended to WHO for their technical support and to MTDF for their efforts in assessing MDR last year. All efforts exerted by FMOH, SMOH, NMDRC, SMDRCs, registrars of obstetrics and gynecology are all appreciated and acknowledged.

\section{References}

[1] WHO, UNICEF, UNFPA, World Bank, 2007. "Maternal Mortality Ratio Falling too Slowly to Meet Goals." Geneva, Switzerland, 20-23.

[2] WHO 1992. "International Statistical Classification of Diseases and Related Health Problems." Instruction Manual. Geneva, Switzerland.

[3] National Committee for the Confidential Enquiry into Maternal Deaths. 1998. Interim Report on the Confidential Enquiry into Maternal Deaths in South Africa. Accessed January 27, 2008, http://www.doh.gov.za/docs/reports/1998/mat_deaths.ht $\mathrm{ml}$.

[4] FMOH-NRHP, UNFPA, WHO, UNICEF, WORLD BANK. 2012. Maternal Death Reviews Report 2009-2011, $1-72$.

[5] Khan, K. S., Wojdyla, D., Say, L., Gülmezoglu, A. M., and Van Look, P. F. 2006. "WHO Analysis of Causes of Maternal Death: A Systemic Review.” Lancet 367 (9516): 1066-74.

[6] Dumont, A., Gaye, A., Bernis, L., Chaillet, N., Landry, A., Delage, J., and Bouvier-Colle, M. 2006. "Facility-Based Maternal Death Reviews: Effects on Maternal Mortality in a District Hospital of Senegal." Bull. World Health Org. 84 (3): 218-24.

[7] Department of Statistics, Ministry of Economics and National Planning and Institute for Resource Development/Macro International, Inc. Sudan. 2007, 
Demographic and Health Survey 1989-1990, Khartoum, Sudan, Ministry of Economics and National Planning and Institute and IRD/Macro, Columbia Maryland. Accessed January 27 , 2008, http://www.measuredhs.com/aboutsurveys/search/metada ta.cfm?surv_id=29\&ctry_id=38\&SrvyTp=type.

[8] Say, L., Chou, D., Gemmill, A., Tunçalp, Ö., Moller, A. B., Daniels, J., Gülmezoglu, A. M., Temmerman, M., and Alkema, L. 2014. "Global Causes of Maternal Death: A WHO Systematic Analysis." Lancet 2 (6): e323-33.

[9] FMOH-NRHP. 2009. Road Map for Reducing Maternal \& Newborn Mortality in Sudan (2010-2015). Khartoum: 15-34.

[10] Bonnar, J. 2000. "Massive Obstetric Hemorrhage."
Baillieres Best Pract. Res. Clin. Obstet. Gynaecol. 14 (1): 1-18.

[11] Potts, M., and Hemmerling, A. 2006. "The Worldwide Burden of Postpartum Haemorrhage: Policy Development Where Inaction Is Lethal." International Journal of Gynecology \& Obstetrics 94 (suppl 2): S116-21.

[12] FMOH-NRHP, UNFPA, WHO, UNICEF, WORLD BANK. 2013. Maternal Death Reviews Report, 1-21.

[13] McCaw-Binns, A., Alexander, S. F., Lindo, J. L., Escoffery, C., Spence, K., Lewis-Bell, K., and Lewis, G. 2007. "Epidemiologic Transition in Maternal Mortality \& Morbidity: New Challenges for Jamaica." International Journal of Gynecology \& Obstetrics 96 (3): 226-32. 